\title{
Can a Virtual Reality Assessment of Fine Motor Skill Predict Successful Central Line Insertion?
}

\author{
Hossein Mohamadipanah, $\mathrm{PhD}^{\mathrm{a}}$, Chembian Parthiban, MS ${ }^{\mathrm{b}}$, Jay Nathwani, $\mathrm{MD}^{\mathrm{a}}$, Drew Rutherford, $\mathrm{MS}^{\mathrm{c}}$, Shannon \\ DiMarco, BA ${ }^{\mathrm{a}}$, Carla Pugh, MD, PhD ${ }^{\mathrm{a}}$
}

\author{
aDepartment of Surgery, School of Medicine and Public Health, University of Wisconsin-Madison \\ 600Highland Avenue, Madison, WI 53726, USA \\ ${ }^{b}$ Department of Mechanical Engineering, College of Engineering, University of Wisconsin-Madison, 3107 \\ Mechanical Engineering Building ,1513 University Avenue, Madison, WI 53706-1572, WI 53706, USA \\ ${ }^{\mathrm{c}}$ Department of Kinesiology, School of Education, University of Wisconsin-Madison \\ 2000 Observatory Dr., Madison, WI 53706, USA
}

Corresponding Author:

Carla M. Pugh, MD, PhD, FACS

Department of Surgery

University of Wisconsin Hospital and Clinics

600 Highland Avenue

Madison, WI 53729

Phone: (608) 262-2147

Fax: (608) 263-2354

pugh@ surgery.wisc.edu 


\section{Introduction}

Evaluation of surgical skills is currently performed using human observation. For example, objective structured assessment of technical skills (OSATS) ${ }^{1}$ is widely used in the surgical education community. While OSATS and other methods are useful, these evaluations are labor-intensive ${ }^{2}$ and require the use of surgical experts who have limited amounts of time for evaluation activities. In addition, observation based assessments are subjective and prone to bias ${ }^{1}$. For instance, the halo effect can influence the evaluation of surgical skills ${ }^{3}$.

In an attempt to address the limitations of observer-based feedback, there is an increase in the amount of research dedicated to non-observer-based (objective) evaluations of surgical skills ${ }^{4-6}$. Advances in sensor technologies and the computer sciences enable this effort and provide a potential means of achieving automated, objective evaluations. For example, a variety of motion metrics have been developed to assess surgical performance by tracking the motion of surgical tools ${ }^{7}$. Also, virtual reality (VR) has been used to educate and assess trainees' psychomotor skills outside the operating room (OR) ${ }^{8}$.

Assessment of psychomotor skills has been conducted by defining motion metrics that have correlations to surgical skills ${ }^{7}$. For instance, in the assessment of pre-defined tasks on a laparoscopic VR simulator, variables such as smoothness, path length, and task execution time have been validated as objective assessment metrics ${ }^{9}$. In another study, a laparoscopic VR simulator was validated as an objective assessment tool assessing fine motor metrics using angular path length ${ }^{10}$ as a measure of skill. Although previous studies have demonstrated validity evidence for objective measures of surgeons' psychomotor skills, a finer relationship between objective metrics and specified procedure steps would provide more explicit guidelines for training and remediation. The majority of the research on motion metrics has focused on motor skills and not decision making, the latter of which is an important part of surgical performance ${ }^{11}$. This is the first time that the metric Idle Time is used to investigate decision making skills in the subclavian central venous catheter (CVC).

The aim of this study is to identify a relationship between the ability of surgical residents to discriminate forces in a VR environment and the use of specific motor movements during needle insertion 
for subclavian CVC. We hypothesize that ability in fine motor discrimination, as assessed in a VR environment will have significant correlations with the gross motor movements during subclavian CVC.

\section{Methods}

Participants

Residents from seven general surgery programs in the United States $(N=43)$ participated in this study. All participants identified themselves as right handed, with exception to one participant who was left handed and one who was ambidextrous. The University of Wisconsin Hospitals and Clinics (UWHC) Institutional Review Board has approved this study.

\section{Motion tracking system and motion metrics}

Each participant was equipped with a head camera that recorded his or her performance during the placement of the subclavian CVC. They were additionally equipped with motion tracking sensors from a Motion Monitor system (Innovative Sport Training, Inc), as shown in Figure 1. These magnetic sensors were affixed to participants' index fingers, thumbs, and wrists in standard positions. The motion tracking system uses sensors that provide six variables, which includes the position and orientation of each sensor. Figure 2 shows examples of position and orientation relative to hand movements. The motion data received by the sensors is filtered by a low-pass second-order Butterworth filter (with a cutoff frequency of $1 \mathrm{~Hz}^{12}$ ) to remove noise.

We used a total of four metric points of interest from the hand movement data while participants were performing the subclavian CVC insertion. "Insertion Time" is defined as the duration of needle insertion into the manikin. "Idle Time" ${ }^{4}$ is defined as the duration of time that the magnitude of velocity of all sensors were less than $0.02 \mathrm{~m} / \mathrm{s}$ when executing needle insertion. "Position Smoothness" is based on the derivative of the position acceleration ${ }^{13}$ and similarly "Orientation Smoothness" is based on the derivative of the orientation acceleration. Greater magnitudes for the smoothness metrics represent greater smoothness. 


\section{Subclavian central line simulation}

Participants were asked to insert a subclavian central line on a simulated hypotensive, tachycardic, and febrile patient. A complete subclavian CVC kit with all the necessary equipment was provided to the participants to perform a subclavian CVC insertion. The simulation model (CentralLineMan System, Simulab Corp, Seattle, WA) had a realistic anatomy of a right upper adult torso. The simulator was draped and prepped for the procedure in a standard surgical fashion. The simulator was placed in Trendelenburg position ( 24 degrees). The participant's view of the scenario is shown in Figure 3 through a head camera. Our particular interest was in needle insertion duration, as proper needle insertion is a crucial subtask for successful subclavian central line placement ${ }^{14}$. Hence, all of the hand movement metrics for this study are calculated only during needle insertion.

\section{Fine motor force discrimination using virtual reality}

As an additional task, the participants were asked to perform a fine motor force discrimination test with the help of a VR haptic device (Force Dimension Omega ${ }^{\circledR}$ ), as shown in Figure 4. The haptic device provides force feedback based on interactions of the operator within a VR environment. The device includes a virtual stylus that can be moved by a participant in the $3 \mathrm{D}$ environment to feel the reaction force of two predefined blocks, namely blocks A and B in Figure 5. Participants are asked to discriminate forces within the two blocks by tapping on them and feeling the force feedback given by the haptic device.

Each trial had a time limit of either 30,20 or 15 seconds to force participants to make a decision regarding their comparison of force for the two blocks. The initial two trials were set to a 30 second time limit to make sure the participant understands the task. Subsequent trials were set to have 15,20 or 30 seconds time limits. We have provided an Appendix (Table 3) with the exact sequencing. Once the participant sampled the force discrimination, he/she indicated which block had the highest force by 
clicking the button on the stylus while the stylus was placed on the respective block. Some trials had blocks with equal forces. In this case, the participant clicked on a designated area between the blocks. Successful discrimination (correctness) was displayed on the screen after every trial and was recorded for offline analysis. Additional details for the force discrimination task in the VR environment is provided in the Appendix. In all trials, the total number of correct force discriminations was labeled "Correct Discrimination", while the total number of wrong force discriminations was labeled "Wrong Discrimination". To make sure that each participant understood the task, the first five trials of each participant were excluded from analysis.

\section{Results}

Table 1 illustrates the correlation between VR force discrimination metrics and the time related metrics in subclavian CVC. The metric "Correct Discrimination" has a negative correlation $(\mathrm{r}=-0.3802$, $\mathrm{p}=0.0119)$ and the metric "Wrong Discrimination" has a positive correlation $(\mathrm{r}=+0.3317, \mathrm{p}=0.0298)$ to the "Insertion Time" metric meaning, persons with better fine motor skills had less insertion time. In addition, the metric "Correct Discrimination" has a negative correlation $(r=-0.4628, p=0.0018)$ to the "Idle Time" metric and the metric "Wrong Discrimination" has a positive correlation $(\mathrm{r}=+0.4108, \mathrm{p}=$ 0.0062) to the "Idle Time" metric. In essence, those persons with excellent fine motor force discrimination have less pauses during needle insertions and those with poor fine motor force discrimination have longer pauses. All of the reported correlation coefficients in this study are Spearman's rho coefficients.

Videos of subclavian CVC indicated that all participants used their right hand for handling the syringe including the left handed and ambidextrous participants. This syringe handling may be the result of simulation scenario setting where the head of the simulator is located on the left side of the participant, shown in Figure 3. There was not an option to stand on the other side. To assess for potential effects, we 
calculated the average of the fine motor metrics from the three sensors on the left hand and the average of the fine motor metrics from the three sensors on the right separately.

Table 2 presents the calculated correlations between VR force discrimination metrics and the position and orientation smoothness in subclavian CVC. The metric "Correct Discrimination" in the VR environment demonstrates positive correlation to the metrics "Positon Smoothness Left Hand" $(\mathrm{r}=$ $+0.3797, \mathrm{p}=0.012)$, "Positon Smoothness Right Hand" $(\mathrm{r}=+0.3683, \mathrm{p}=0.0151)$, "Orientation Smoothness Left Hand" $(\mathrm{r}=+0.3269, \mathrm{p}=0.0324)$, and "Orientation Smoothness Right Hand" $(\mathrm{r}=$ $+0.4135, \mathrm{p}=0.0059$ ) from subclavian CVC simulation. In addition, the metric "Wrong Discrimination" in the VR environment did not correlate $(\mathrm{p}>0.05)$ to any metric from subclavian CVC simulation.

We set $p<0.05$ as the significant level in our analysis, however to have a more conservative analysis we used Bonferroni importance level $p<\left(\frac{0.05}{6}=0.0083\right)$ (as there are six motion metrics in this study). By considering this significant level in Table 1, the metric "Correct Discrimination" has a negative significant correlation and the metric "Wrong Discrimination" has a positive significant correlation $(p<0.0083)$ to the "Idle Time" metric. In Table 2, the metric "Correct Discrimination" has shown significant positive correlation $(\mathrm{p}<0.0083)$ to the metrics "Orientation Smoothness Right Hand".

\section{Discussion}

This study sought to validate the relationship between fine motor force discrimination in a VR environment and gross motor metrics in the insertion of a subclavian CVC on a simulator. Our analysis reveals that participants who had less insertion time made a higher number of correct decisions and lower number of wrong decisions during the VR discrimination task. Also, correlations between the metric "Idle Time" and the "Correct Differentiation" and "Wrong Differentiation" in Table 1 implies that participants with more ability in discriminating forces in the VR environment had less amount of uncertainty during their needle insertion. The findings in Table 2 show that smoother position and orientation movements of the hands imply a certain level of underlying proficiency in needle insertion ability during subclavian 
CVC. As all of the participants held the syringe with their right hands, the findings in Table 1 and Table 2 imply that orientation smoothness of the syringe holding hand and "Idle Time" are the most informative $(\mathrm{p}<0.0083)$ fine motor metrics in subclavian CVC procedure.

Findings in this study also indicate that haptic sensation is an important motor skill that could affect performance of needle insertion in subclavian CVC. Those who have poor haptic sensation may miss important cues when passing through the chest wall tissues. For instance, when the needle first hits the clavicle, the participant should put light pressure on the needle with their opposite hand for guidance of the needle into the subclavian vein. Those who fail to feel the needle hit the clavicle may continue to exert force, causing tissue damage and undue pain for the patient. In addition, these actions may result in an unsuccessful venous puncture.

Moreover, we are going to study the effect of haptic sensation skills on the other open and laparoscopic surgical scenarios, including anastomosis bowel repair and laparoscopic ventral hernia repair, in our future studies. In addition, VR simulators, such as the MIST-VR ${ }^{15}$, have been widely used and studied in surgical education and have been successful in showing that training in a virtual environment can reduce errors in the operating room. Consequently, there is an opportunity to use VR to test specific surgical and psychomotor skills as these systems can mimic a wide range of haptic sensations and automatically record detailed performance metrics based on human computer interaction.

While "Smoothness" and "Idle Time" metrics have shown great potential as motion metrics in evaluating subclavian $\mathrm{CVC}$, more work needs to be done to translate these metrics into learning objectives and skill exercises for trainees. For example, telling a resident that their performance was not smooth enough is not specific enough to improve performance.

There are several other limitations in this study. One is the small sample size that we plan to address by conducting additional data collections. The other limitation was the nature of participation, which was on a voluntary basis of the general surgery residents. Therefore, the results of this study exclude the residents who did not accept to be a volunteer and participate in this study. Future work will also focus on assessing the difference between the VR force discrimination metrics and global ratings 
obtained by experts for subclavian CVC. This will allow additional validation of our findings and an assessment of how VR assessments could be used to guide fine motor skills training for residents as faculty time is limited and may be less objective and instructive.

Acknowledgements 
Funding for this study came from the Department of Defense grant \#W81XWH-13-1-0080 entitled "Psycho-Motor and Error Enabled Simulations Modeling Vulnerable Skills in the Pre-Mastery Phase Medical Practice Initiative Procedural Skill Decay and Maintenance (MPI-PSD)". 


\section{Figure Legends}

\section{Figure 1.}

Sensors attached to a participant's index finger, thumb, and wrist.

\section{Figure 2.}

Two types of hand movement; (Top) position movement (Bottom) orientation movement.

\section{Figure 3.}

The participant's view of the subclavian CVC scenario.

\section{Figure 4.}

The haptic device operated by a participant during data collection.

\section{Figure 5.}

Screenshot of the force discrimination task in the virtual environment. Shows blocks A and B of different forces being rendered and the virtual stylus (blue). 


\section{Appendix:}

In the VR environment, each participant was provided with 19 trials, where the object forces were set between (1.45-13.05) N/mm which corresponds to $10 \%$ and $90 \%$ of the maximum closed loop force handled by the device, which is $14.5 \mathrm{~N} / \mathrm{mm}$. The object force selections were based on a randomly repeated combination of 16 different trial conditions, which is shown in Table 3 . The effective force discrimination between objects $A$ and $B$ shown in Figure 5 was between $(0$ and 8.7$) \mathrm{N} / \mathrm{mm}$, where 0 means the objects had equal force and $8.7 \mathrm{~N} / \mathrm{mm}$ was the maximum difference in force amongst all the trials. 


\section{Table Legends}

Table 1.

The correlation between force discrimination metrics in VR and the time related metrics in subclavian CVC.

Table 2.

The correlation between the fine motor metrics in subclavian CVC and VR force discrimination metrics.

Table 3.

Shows the summary of the trial condition parameters and its frequency distribution as used for the 43 participants. The 19 trials were a combination of the 16 Trial Conditions that was randomly assigned to the participants in the form of 3 combination files, called combo 1, combo 2, and combo 3. Each of combo 1 and combo 2 were used for 15 , and combo 3 was used for 13 participants out of total 43 participants. 


\section{References}

[1] Martin JA, Regehr G, Reznick R, et al. Objective structured assessment of technical skill (OSATS) for surgical residents. British Journal of Surgery. 1997;84:273-278.

[2] Sidhu RS, Grober ED, Musselman LJ, Reznick RK. Assessing competency in surgery: Where to begin? Surgery. 2004;135:6-20.

[3] Wanzel KR, Ward M, Reznick RK. Teaching the surgical craft: From selection to certification.

Current Problems in Surgery. 2002;39:583-659.

[4] D'Angelo A-LD, Rutherford DN, Ray RD, et al. Idle time: an underdeveloped performance metric for assessing surgical skill. The American Journal of Surgery. 2015;209:645-651.

[5] Dosis A, Aggarwal R, Bello F, et al. Synchronized video and motion analysis for the assessment of procedures in the operating theater. Archives of Surgery. 2005;140:293-299.

[6] Datta V, Mandalia M, Mackay S, Chang A, Cheshire N, Darzi A. Relationship between skill and outcome in the laboratory-based model. Surgery. 2002;131:318-323.

[7] Oropesa I, Sánchez-González P, Lamata P, et al. Methods and Tools for Objective Assessment of Psychomotor Skills in Laparoscopic Surgery. Journal of Surgical Research. 2011;171:e81-e95.

[8] Aggarwal R, Moorthy K, Darzi A. Laparoscopic skills training and assessment. British Journal of Surgery. 2004;91:1549-1558.

[9] Pellen MGC, Horgan LF, Barton JR, Attwood SE. Construct validity of the ProMIS laparoscopic simulator. Surgical Endoscopy. 2008;23:130-139.

[10] Dongen KW, Tournoij E, Zee DC, Schijven MP, Broeders IAMJ. Construct validity of the LapSim: Can the LapSim virtual reality simulator distinguish between novices and experts? Surgical Endoscopy. 2007;21:1413-1417.

[11] Pugh C, Plachta S, Auyang E, Pryor A, Hungness E. Outcome measures for surgical simulators: Is the focus on technical skills the best approach? Surgery. 2010;147:646-654.

[12] Falk V. Manual control and tracking-a human factor analysis relevant for beating heart surgery. The Annals of Thoracic Surgery. 2002;74:624-628.

[13] Hogan N, Sternad D. Sensitivity of Smoothness Measures to Movement Duration, Amplitude and Arrests. Journal of motor behavior. 2009;41:529-534.

[14] McGee DC, Gould MK. Preventing Complications of Central Venous Catheterization. New England Journal of Medicine. 2003;348:1123-1133.

[15] Maithel S, Sierra R, Korndorffer J, et al. Construct and face validity of MIST-VR, Endotower, and CELTS. Surgical Endoscopy And Other Interventional Techniques. 2005;20:104-112. 

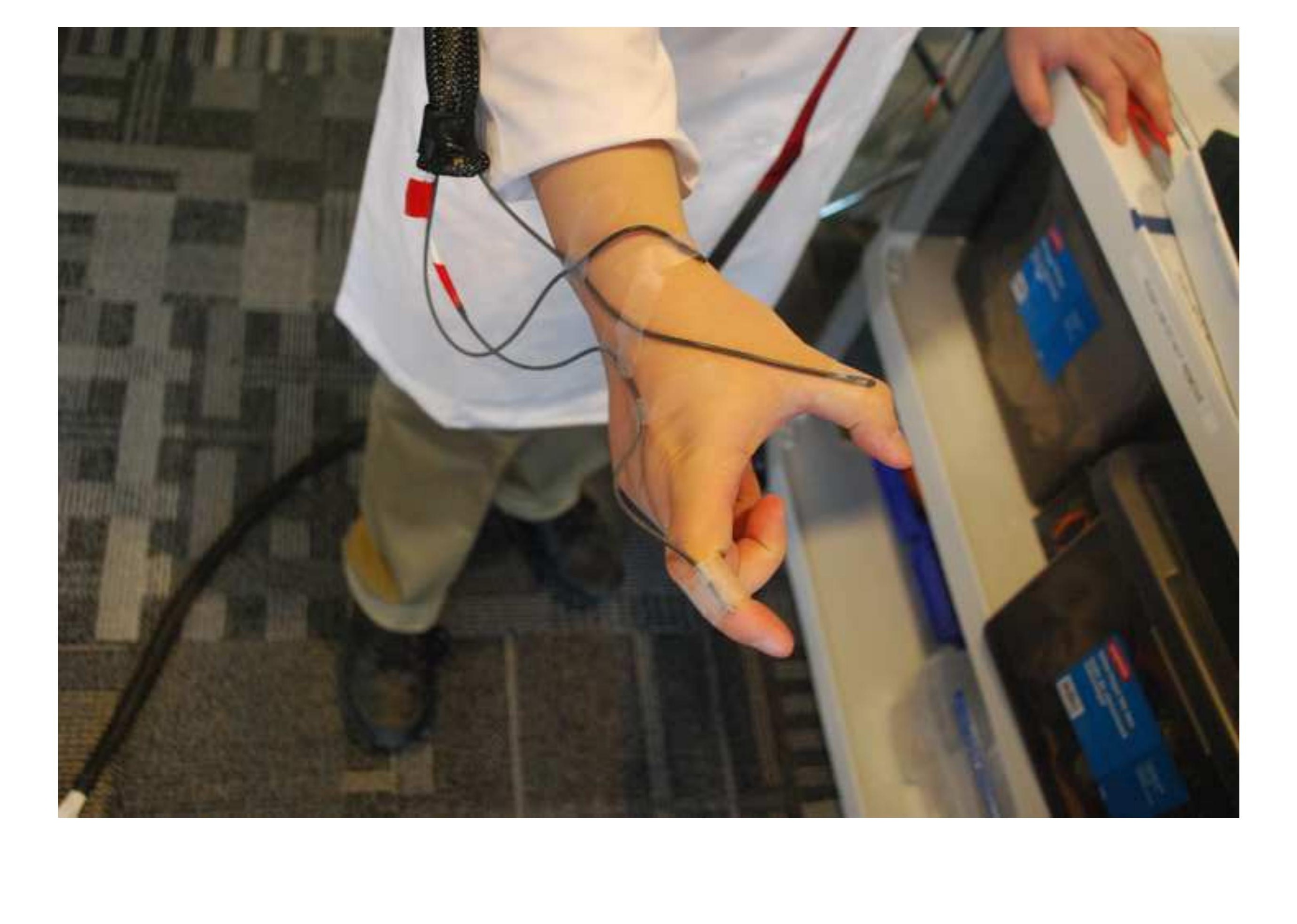


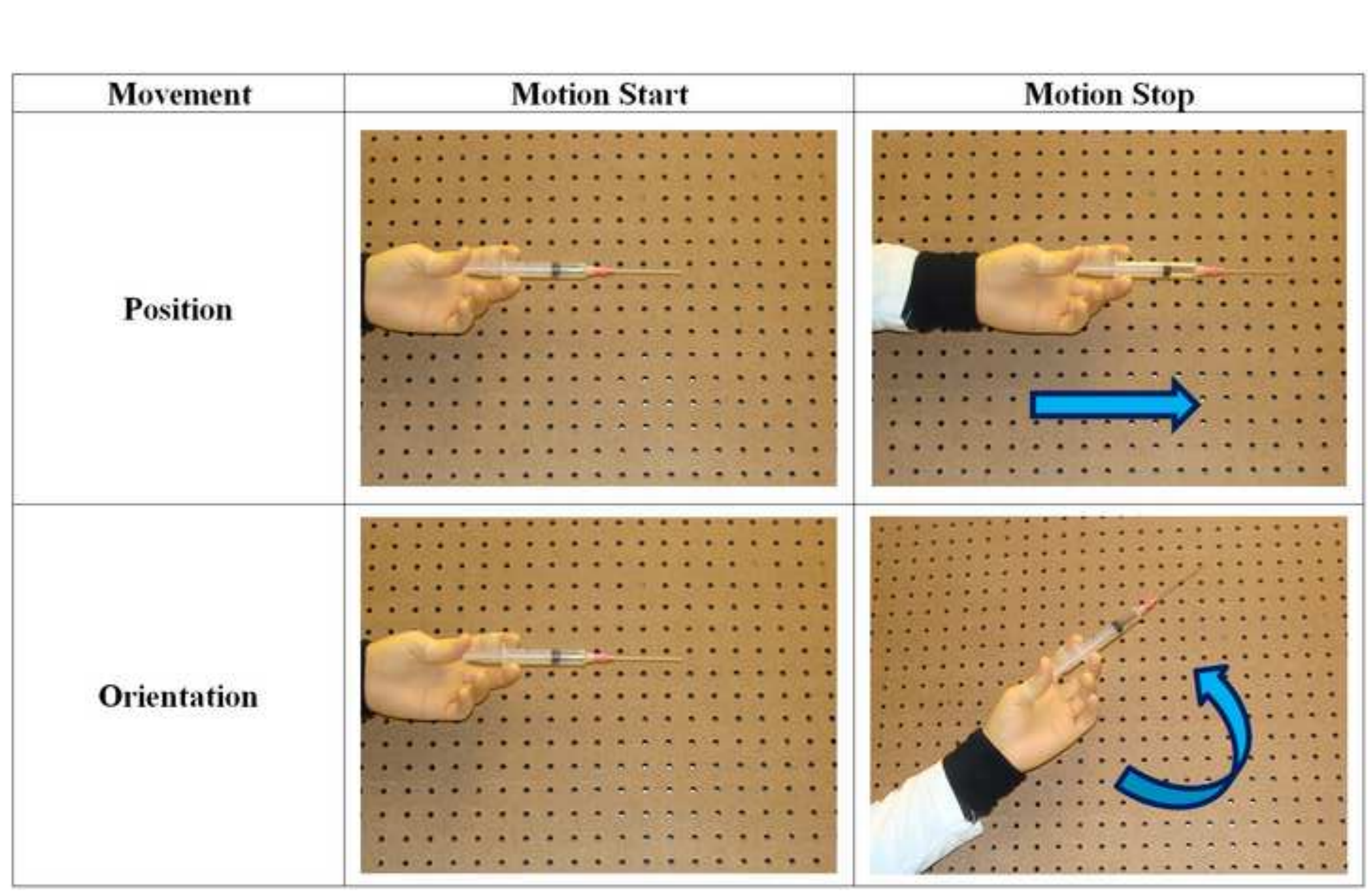
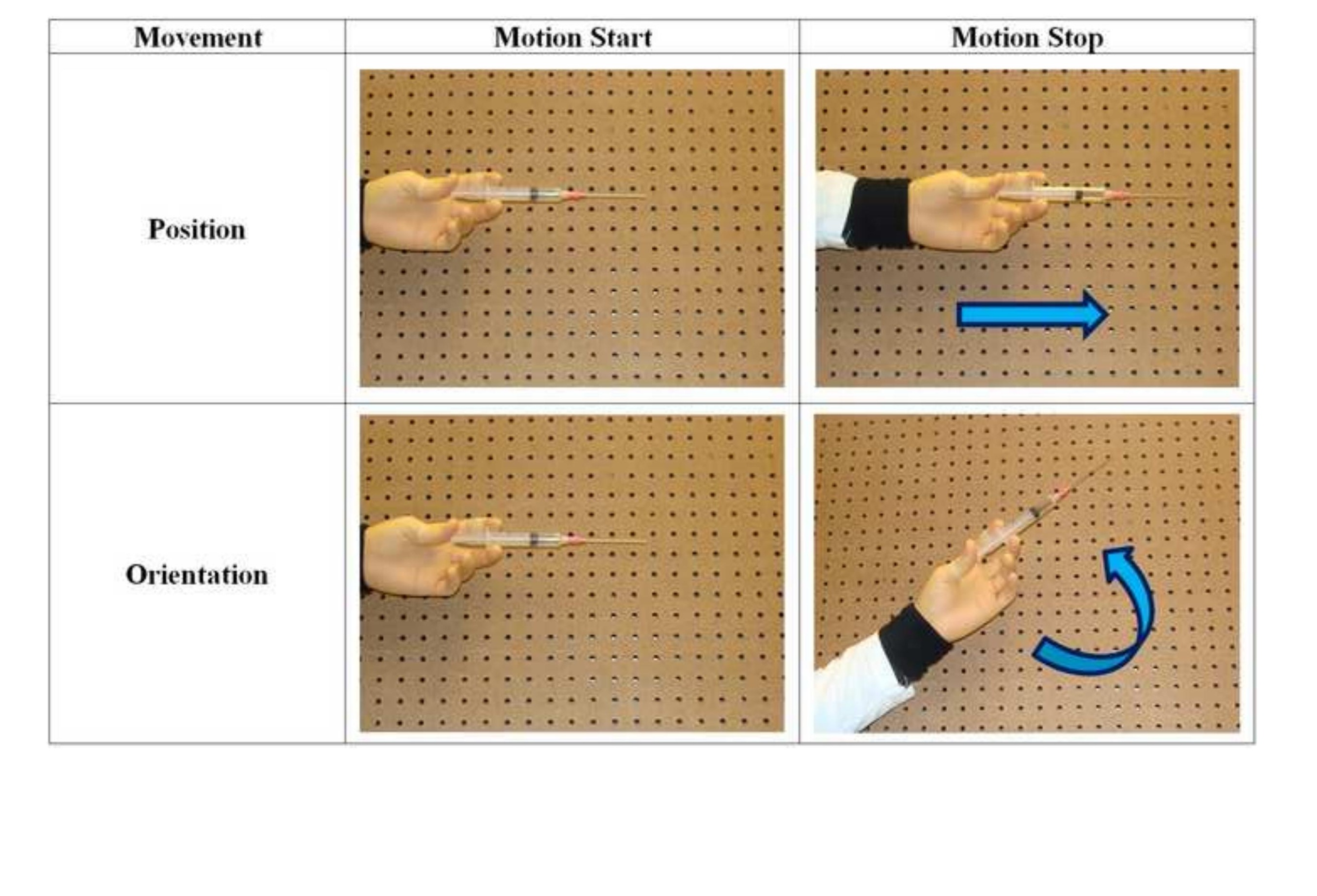

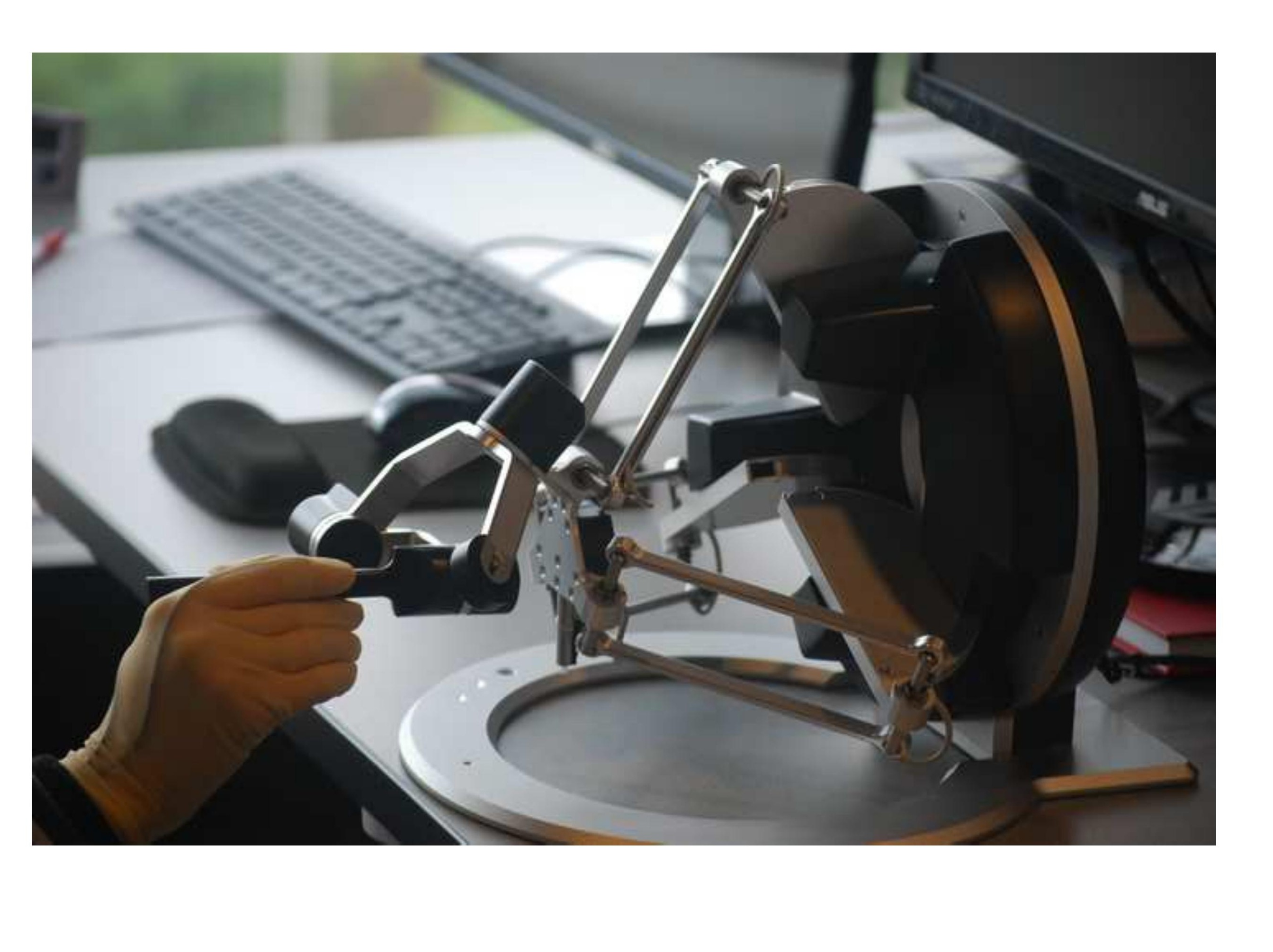
- Move stylus into the start sphere to start. Strike against the two objects that appear to find which feels stiffer

- Select the stiffer block by cticking home bunton by just staying on the respective side (green backgrount denotes selection)

- If stiffnesses feel the same click by staying in between blocks. Select before trial times out (Timer-shown on the left)

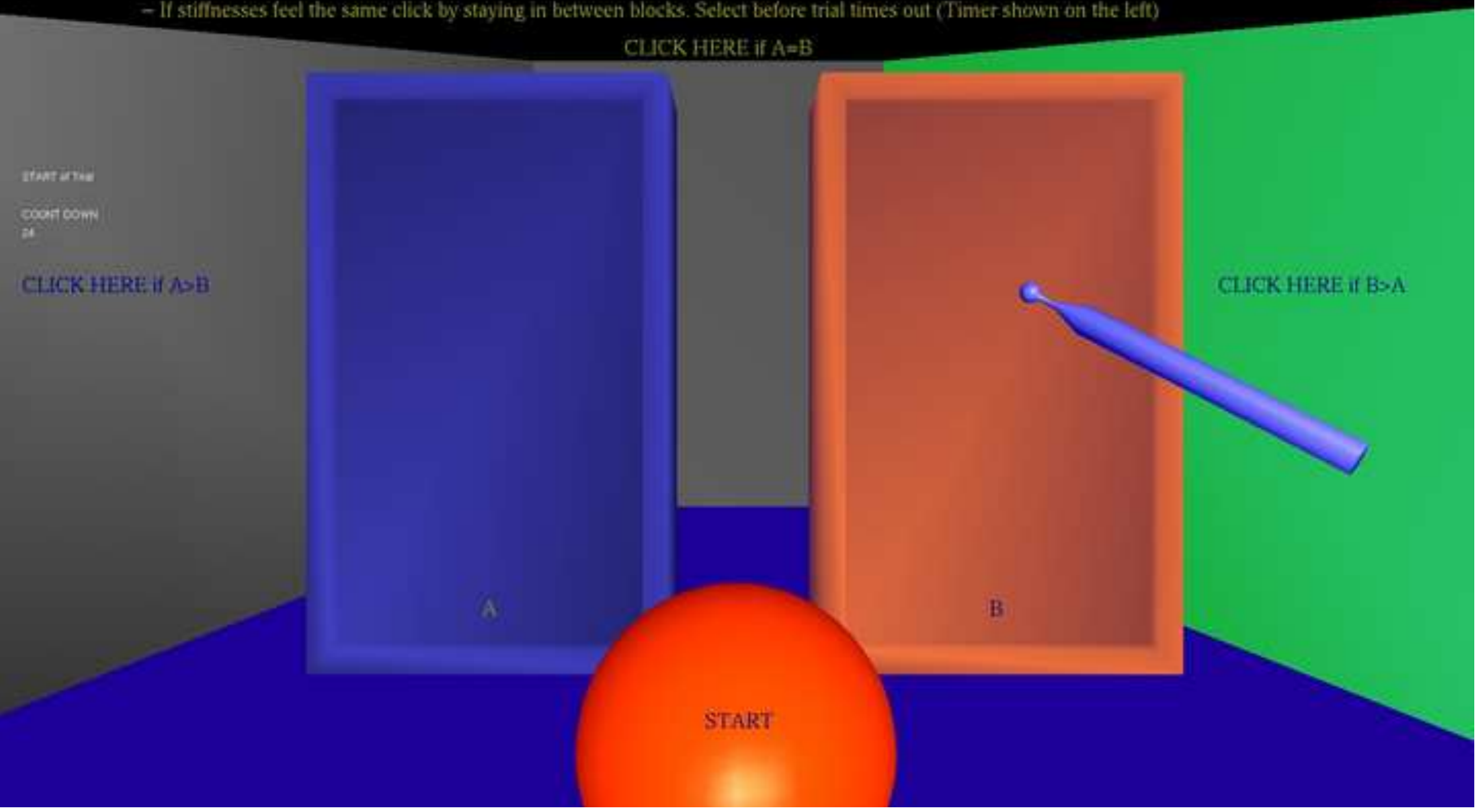




\begin{tabular}{|c|c|c|c|}
\hline Metric of Subclavian Central Line & Metric of Force Discrimination in VR & $\boldsymbol{R}$ & $\boldsymbol{P}$ value \\
\hline \multirow{2}{*}{ Insertion Time } & Correct Discrimination & $\mathbf{- 0 . 3 8 0 2}$ & $\mathbf{0 . 0 1 1 9}$ \\
\cline { 2 - 4 } & Wrong Discrimination & $\mathbf{+ 0 . 3 3 1 7}$ & $\mathbf{0 . 0 2 9 8}$ \\
\hline \multirow{2}{*}{ Idle Time } & Correct Discrimination & $\mathbf{- 0 . 4 6 2 8}$ & $\mathbf{0 . 0 0 1 8}$ \\
\cline { 2 - 4 } & Wrong Discrimination & $\mathbf{+ 0 . 4 1 0 8}$ & $\mathbf{0 . 0 0 6 2}$ \\
\hline
\end{tabular}




\begin{tabular}{|c|c|c|c|}
\hline Metric of Subclavian Central Line & Metric of Force Discrimination in VR & $\boldsymbol{R}$ & P value \\
\hline \multirow{2}{*}{$\begin{array}{c}\text { Position Smoothness } \\
\text { Left Hand }\end{array}$} & Correct Discrimination & +0.3797 & $\mathbf{0 . 0 1 2 0}$ \\
\hline & Wrong Discrimination & -0.2568 & 0.0965 \\
\hline \multirow{2}{*}{$\begin{array}{l}\text { Position Smoothness } \\
\text { Right hand }\end{array}$} & Correct Discrimination & +0.3683 & 0.0151 \\
\hline & Wrong Discrimination & -0.2113 & 0.1738 \\
\hline \multirow{2}{*}{$\begin{array}{c}\text { Orientation Smoothness } \\
\text { Left Hand }\end{array}$} & Correct Discrimination & +0.3269 & 0.0324 \\
\hline & Wrong Discrimination & -0.2024 & 0.1931 \\
\hline \multirow{2}{*}{$\begin{array}{c}\text { Orientation Smoothness } \\
\text { Right hand }\end{array}$} & Correct Discrimination & +0.4135 & 0.0059 \\
\hline & Wrong Discrimination & -0.2238 & 0.1492 \\
\hline
\end{tabular}




\begin{tabular}{|c|c|c|c|c|c|c|c|}
\hline $\begin{array}{c}\text { Trial } \\
\text { Condition }\end{array}$ & $\begin{array}{c}\text { Time } \\
\text { Limit } \\
{[\text { Seconds] }}\end{array}$ & $\begin{array}{c}\text { Force of } \\
\text { Block A } \\
{[\mathbf{N} / \mathbf{m m}]}\end{array}$ & $\begin{array}{c}\text { Force of } \\
\text { Block B } \\
{[\mathbf{N} / \mathbf{m m}]}\end{array}$ & $\begin{array}{c}\text { Force } \\
\text { Difference } \\
{[\mathbf{N} / \mathbf{m m}]}\end{array}$ & $\begin{array}{c}\text { Combo 1 } \\
\text { Frequency }\end{array}$ & $\begin{array}{c}\text { Combo 2 } \\
\text { Frequency }\end{array}$ & $\begin{array}{c}\text { Combo 3 } \\
\text { Frequency }\end{array}$ \\
\hline 1 & 30 & 8.700 & 11.600 & 2.900 & 2 & 2 & 2 \\
\hline 2 & 20 & 10.150 & 5.800 & 4.350 & 1 & 1 & 0 \\
\hline 3 & 30 & 7.250 & 4.350 & 2.900 & 1 & 1 & 1 \\
\hline 4 & 30 & 5.800 & 8.700 & 2.900 & 1 & 2 & 1 \\
\hline 5 & 15 & 8.700 & 8.700 & 0.000 & 1 & 1 & 2 \\
\hline 6 & 15 & 4.350 & 2.900 & 1.450 & 1 & 2 & 3 \\
\hline 7 & 20 & 13.050 & 13.050 & 0.000 & 1 & 1 & 0 \\
\hline 8 & 15 & 2.175 & 2.175 & 0.000 & 1 & 1 & 0 \\
\hline 9 & 20 & 4.350 & 11.600 & 7.250 & 1 & 1 & 4 \\
\hline 10 & 15 & 13.050 & 5.800 & 7.250 & 2 & 1 & 1 \\
\hline 11 & 20 & 2.900 & 1.450 & 1.450 & 2 & 1 & 2 \\
\hline 12 & 15 & 1.450 & 2.900 & 1.450 & 1 & 1 & 1 \\
\hline 13 & 20 & 2.175 & 3.625 & 1.450 & 1 & 1 & 1 \\
\hline 14 & 15 & 6.525 & 7.250 & 0.725 & 1 & 1 & 0 \\
\hline 15 & 20 & 1.450 & 10.150 & 8.700 & 1 & 1 & 0 \\
\hline 16 & 15 & 11.600 & 12.325 & 0.725 & 1 & 1 & 1 \\
\hline
\end{tabular}

